\title{
Umbilical Discharge and Its Management in Adults-Rare Presentatation
}

\author{
Dr vikas Sharma
}

\section{Abstract}

Objectives: Current study has been conducted to find out the varied presentation of umbilical discharge in adults and their outcome after appropriate management.

Study design : The study included the patients (29) admitted and diagnosed as a case of umbilical discharge in 5 years period from January, 2005 to December 2009. A total of 29 cases were admitted and 27 underwent surgery.

Results: Maximum number of cases of umbilical discharge in adults was due to patient urachal fistulas. Other causes include post laproscopic surgery umbilical discharge, pilonidal sinus, infection due to hair follicals and foreign bodies and non-specific acute and chronic inflammation and abscess of umbilicus .

Conclusion: Umbilical discharge is rare in adults \& it should be thoroughly \& swiftly evaluated And Failure to diagnose many result in poor outcome.

\section{Introduction}

Development of umbilicus is one of the most complex embryological process in human beings and due to this, it is subjected to a large number of malformations.

Majority of these malformations manifests in the neonatal period. But rarely these congenital malformation may present in young adult age group and they most commonly present with discharge through umbilicus.

In this study of 29 cases of umbilical discharge in adults, the most common cause that we came across is patent urachus, accounting for a total of nearly $50 \%$.

\section{Embryology:}

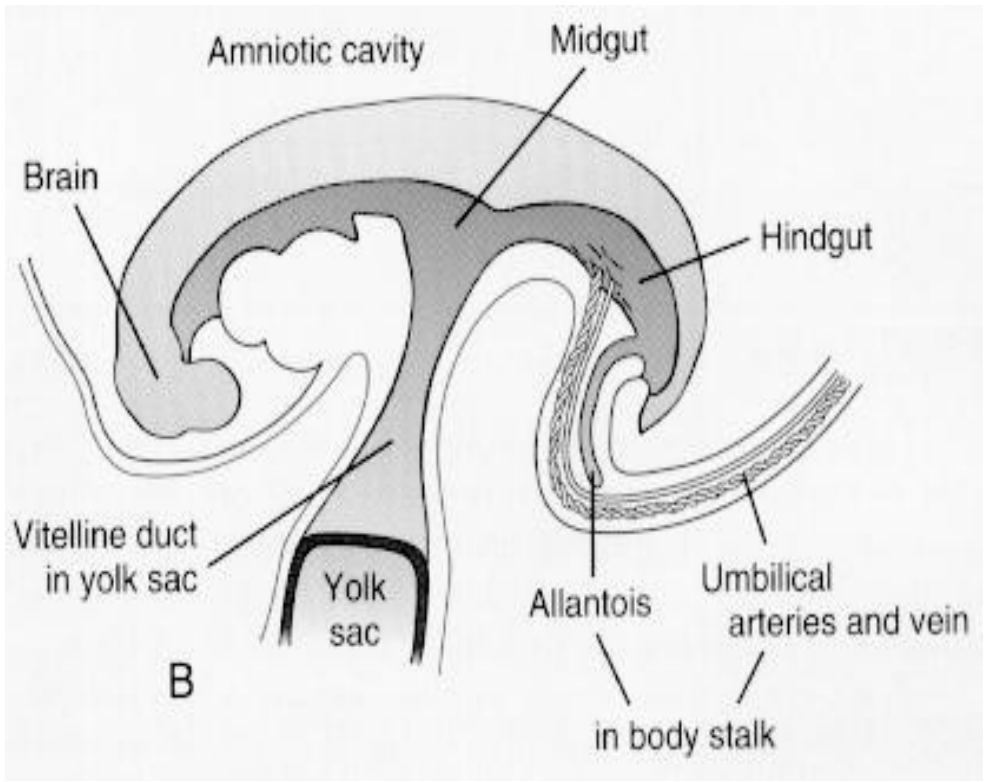

The embryonic disc elongates and bulges towards aminon and the 2 ends ( head and tail) comes close together and encloses a part of yolk sac within the endodermal lining of disc. This part of yolk sac forms the gut ( foregut, midgut and hindgut ). The narrow part of yolk sac between 2 ends of endoderm form V-I duct and rest of yolk sac disappears ${ }^{1}$. 

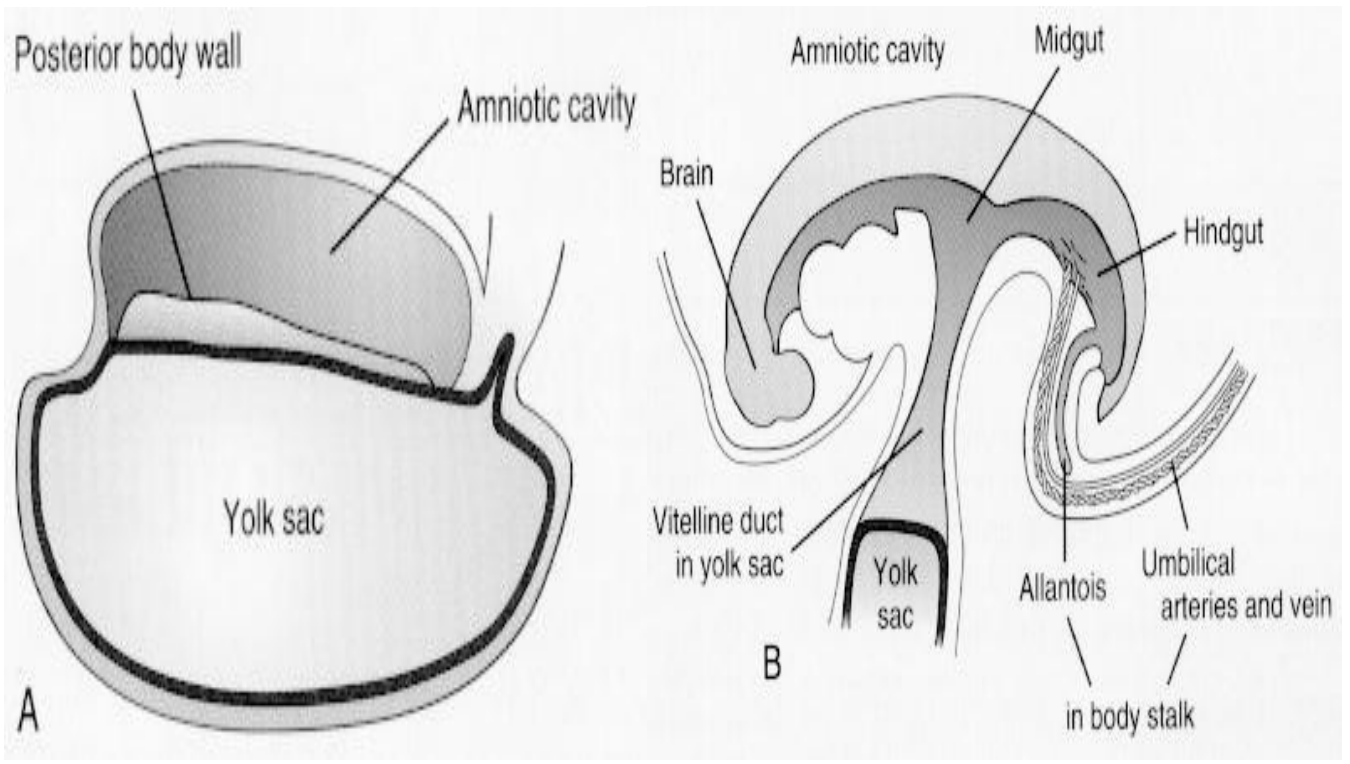

The allantios begins as a projection of endodermal layer of embryonic disc into the extraembryonic mesoderm. Later as the disc elongates, this allantois fuses into hind gut and projects towards umbilical cord ${ }^{1,12}$.

Normally both V.I. duct ( which connects gut to umbilicus ) and urachus ( which connects U.B. to umbilicus ) gets obliterated. But these may not get obliterated and give rise to varied presentations:-

\section{$\underline{\text { Urachus }}$}

1. Patent urachus

2. Urachal cyst

3. Umbilical urachal

Sinus

4. Vesico-urachal diverticulum

\section{V.I. duct}

1. Vitelline fistula

2. Vitelline cyst

3. Vitelline cord

4. Meckel's diverticulum

5. Meckel's diverticulum with

Vitelline cord

6 . Vitelline umbilical sinus

\section{Material And Methods}

This study consists of 29 cases of young adult patients in the age group of $12-60$ yrs with umbilical discharge ( 25 males and 4 females) admitted in surgical wards and attended in surgery OPD in various centres from January 2005 to December 2009. Patient's details, particularly age and clinical presentation were observed.

\section{Plan of work:}

A detailed history was obtained for the type of discharge in respect of Amount, colour, odour and nature- whether discharge is pus, fecal matter, urine, serus, serosanguinous or blood. Time and mode of onset, duration since its appearance. Whether associated with some swelling. H/o bladder and bowel habbits and related symptoms like pain, vomiting, any problem with micturition were asked.

\section{Physical Examination :}

A thorough general and systemic examination was done to determine

1. Exact site of pathological discharge.

2. Whether anomaly associated with some other congenital anomaly or disease.

3. Any other specific associated problems like pyrexia, dehydration and septicemia.

\section{Investigations :}

Lab investigations

- $\quad \mathrm{Hb}, \mathrm{TLC}, \mathrm{DLC}, \mathrm{ESR}$

- BT, CT

- Urine : Routine / Microscopic

- Urine : Culture sensitivity 
- Discharge : Culture sensitivity \& cytology

- $\quad$ RBS, S. Urea, S. Creatinine

- H-P Examination

Imaging Techniques:

- X-ray abdomen

- BMFT

- USG, CT scan

- Fistulogram, Sinogram

- $\mathrm{MCU}$

\section{Results}

Final diagnosis of the 29 cases admitted with umbilical discharge was as follows:

\begin{tabular}{|l|l|l|l|}
\hline Diagnosis & $\begin{array}{l}\text { No. of patients } \\
\text { (out of 29) }\end{array}$ & Percentage & Symptom \\
\hline Patient Urachus & 15 & $51.72 \%$ & Urine discharge \\
\hline Post laproscopic surgery & 3 & $10.34 \%$ & Serous/purulent discharge \\
\hline Umbilical hernia with ulceration & 3 & $10.34 \%$ & Sero-purulent discharge \\
\hline Umblical Abscess & 2 & $6.89 \%$ & Pus discharge \\
\hline Vitelline umbilical sinus & 2 & $6.89 \%$ & Serous discharge \\
\hline Recurrent folliculitis & 2 & $6.89 \%$ & $\begin{array}{l}\text { Inflammation \&Pus } \\
\text { discharge }\end{array}$ \\
\hline Advanced Ca GB & 1 & $3.44 \%$ & $\begin{array}{l}\text { Growth with sero-sanguinous } \\
\text { discharge }\end{array}$ \\
\hline Pilonidal sinus & 1 & $3.44 \%$ & Itching\&discharge \\
\hline
\end{tabular}

- Of the 29 cases, 27 were managed operatively and 2 were managed conservatively

Patients were treated as follows:-

\begin{tabular}{|l|l|l|}
\hline Sl.No. & Diagnosis & Treatment \\
\hline 1. & Patent urachus(15 patients) & $\begin{array}{l}\text { All patients treated by complete excision of tracts with a slip of } \\
\text { dome of bladder and dome of bladder was repaired in } 2 \text { layers }\end{array}$ \\
\hline $2 ., 12,14$
\end{tabular}

-Most common organism isolated by culture sensitivity was staph aureus.

\section{Discussion}

- There is no such study conducted in North India as far as umbilical discharge is concerned in adults.

Since umbilical discharge in adults is an uncommon problem. This disease should be taken seriously to understand \& treat it in a better way.

This study shows that umbilical discharge in adults could just be the tip of an iceberg as it could reveal later on a wide spectrum of underlying pathology , the diagnosis of which must be arrived at and treated properly.

In this study most of the cases were managed operatively with uneventful post operative period.

Imaging modality like sonogram,fistulogram,USG \& CT and lab modality like Discharge ( culture and sensitivity) proved very useful in management of patient's with umbilical discharge in adults in this study.

Frequency of port-site infection (PSI) in cases of laparoscopic cholecystectomies reusing disposable ports-Record of all patients undergoing laparoscopic cholecystectomy in Surgical A Unit; LRH,U.K between 2007-1994 was retrospectively analyzed for PSI. Out of 300 cases, 6 cases were converted to open cholecystectomy and excluded from the study. All patients were admitted a day before surgery and were given 3 shots of ceftriaxone $1 \mathrm{gm}$ (Inj. Rocephin, Roche, UK); one before and two after the surgery. The patients were monitored for port site using standard National Nosocomial Infections Surveillance (NNIS) System definitions given by the Centers for Disease Control and Prevention (CDC). All infected wounds were treated by local 
washes and oral antibiotics. Out of 294 cases, 17 (5.78\%) developed PSI. Out of these 17 infected cases 12 (70.5\%) had superficial infection while $5(29.4 \%)$ had deep surgical site infection (SSI). Epigastric port-site was infected in $15(88.2 \%)$ cases followed by the umbilical port-site in $2(11.8 \%)$ cases. Two (11.8\%) patients with port-site infection had operation lasting $<1$ hour while in $15(88.2 \%)$ cases the surgery lasted for $>1 \mathrm{hr}$. Main operative findings were acute cholecystitis in 7 (41.1\%) patients, empyema gall bladder in 4 (23.5\%), adhesions in 3 (17.6\%). Conclusion: Laparoscopic cholecystectomy is associated with a low risk of PSI, which in most cases is only superficial and responds to local measures. It is most commonly the trocar site of gall bladder extraction that is infected. ${ }^{15}$

As the horizon of laproscopic surgery increased in the patients of abdominal malignancies the incidence of port site metastasis ${ }^{16}$ was found to be $0.5 \%-13 \%$. In our study we reported a case of advanced adenocarcinoma GB presenting with umbilical discharge.(3.44\%)

\section{Conclusion}

Following conclusion can be drawn from present study:

- Umbilical discharge in adults is a very rare finding. In our study of 5 years duration,conducted at three centers, on umbilical discharge in adults, patent urachus(51.72\%) is the most common cause followed by post laproscopic surgical site infection(10.34\%) \& umbilical hernia with ulceration(10.34\%).

- Umbilical discharge in any age should be swiftly evaluated. Failure to diagnose may result in increased morbidity \& poor outcome.

- The history and lab. Studies may not enable the clinician to completely narrow the D/D and imaging may be fundamental in securing a timely diagnosis.

- USG, CT scan, sinogram \& fistulogram are very sensitive in detecting urachral remnants, but sonography maintains a pivotal role.

- A very sincere and a team approach with involvement of various departments like surgery,pathology,microbiology and radiodiagnosis, is must for favourable outcome.

\section{References:}

[1]. I.B.Singh .Textbook of Human embryology, development of embryonic disc,vitello-instentinal duct \& urachus

[2]. Kosloske AM, Bartow SA. Debridement of periumbilical necrotizing fasciitis: importance of excision of the umbilical vessels and urachal remnant. J Pediatr Surg. Jul 1991;26(7):808-10. [Medline].

[3]. Hall DE, Roberts KB, Charney E. Umbilical hernia: what happens after age 5 years?. J Pediatr. Mar 1981;98(3):415-7. [Medline].

[4]. Stroup SP, Thoman DS. A naval surgeon's approach to the draining umbilicus. J Laparoendosc Adv Surg Tech A. 2007;17(5):645648. doi: 10.1089/lap.2006.0198. [PubMed] [Cross Ref]

[5]. Eryilmaz R, Sahin M, Okan I, Alimoglu O, Somay A. Umbilical pilonidal sinus disease: predisposing factors and treatment. World J Surg. 2005;29:1158-1160. doi: 10.1007/s00268-005-7895-9. [PubMed] [Cross Ref]

[6]. El-Bakry AA. Discharging umbilicus. Saudi Med J. 2002;23(9):1099-1100. [PubMed]

[7]. Molderez CM, Wouters KB, Bergsmans GB, Michiels GK. Umbilical discharge: a review of 22 cases. Acta Chir Belg. 1995;95(3):166-169. [PubMed]

[8]. Steinhauser G. The nature of navel fluff. Med Hypotheses. 2009;72(6):623-625. doi: 10.1016/j.mehy.2009.01.015. [PubMed] [Cross Ref]

[9]. Begg R.D.: The urachus: Its anatomy, histology and development. J.Anat.64:170,1930.

[10]. Bergglas, B. Genesis and therapy of umbilical hernia. Arch.F.Gynac, 152:214,1933.

[11]. Carter, Franklin R.: Umblical hernia: Types and treatment,arch.Ped.49:622,1932.

[12]. Cullen T.S.: Embryology,Anatomy, and disease of umbilicus together with disease of the urachus. Philadelphia, W.B. Saunders Co.,1916, pp. 351-372.

[13]. Jackson O.F., Muglen L.H.: Umblical hernia: A retrospective study. Calif Med. 113:8, 1970.

[14]. Nix, J.T., J.G. Menville, M.Albert, and D.L. Wendt: Congenital patent urachus. J. Urol. 79:264,1958.

[15]. The frequency of port-site infection in Laparoscopic Cholecystectomies J Postgrad Med Inst Jan - Mar 2008;22(1):66-70.

[16]. Lady Reading Hospital, Peshawar. Fornar P Port metastasis, Fact or fiction urology, 2002, Mar, 41(2). 113-9. 